\title{
Prevalencia y Características Morfométricas del Foramen Mastoideo y Vena Emisaria Mastoidea en Población Mexicana
}

\author{
Prevalence and Morphometric Characteristics of Mastoid Foramen \\ and Mastoid Emmisary Vein in the Mexican Population
}

\begin{abstract}
Alejandra Nohemí Hernández-Rodríguez*; Salvador Galindo-de León*; Rodolfo Morales-Avalos*;
María del Carmen Theriot-Girón**; Oscar de la Garza-Castro* Rodrigo E. Elizondo-Omaña* \& Santos Guzmán-López*
\end{abstract}

HERNÁNDEZ-RODRÍGUEZ, A. N.; GALINDO-DE LEÓN, S.; MORALES-AVALOS, R.; THERIOT-GIRÓN, M. C.; DE LA GARZA-CASTRO, O.; ELIZONDO-OMAÑA, R. E. \& GUZMÁN-LÓPEZ, S. Prevalencia y características morfométricas del foramen mastoideo y vena emisaria mastoidea en población Mexicana. Int. J. Morphol., 32(2):395-398, 2014.

RESUMEN: Las descripciones anatómicas del foramen mastoideo (FM) y la vena emisaria mastoidea (VEM) son escasas en la literatura. Dicho conocimiento es fundamental para realizar un abordaje quirúrgico de la fosa craneal posterior o región mastoidea. El objetivo del estudio fue determinar la prevalencia, variantes y características morfométricas del FM y VEM en población mexicana. Se evaluaron 176 hemicráneos por dos observadores independientes cegados el uno del otro, se determinaron la prevalencia, número y características morfométricas. La prevalencia del FM entre los dos lados fue de $75 \%$ con una mayor frecuencia de forámenes del lado izquierdo comparado con el lado derecho; respecto al número de forámenes se encontraron 108 hemicráneos con un foramen único, 22 con doble foramen y 2 con triple foramen. La prevalencia del FM y VEM fue de 75\%, siendo la variante única la más frecuente.

PALABRAS CLAVE: Foramen mastoideo; Vena emisaria mastoidea; Prevalencia; Características morfométricas; México.

\section{INTRODUCCIÓN}

El foramen mastoideo (FM) es un foramen inconstante ubicado en la superficie posterior del hueso temporal, su contenido corresponde a la vena emisaria mastoidea (VEM); su relevancia anatómica radica en que representa un punto de anastomosis venosa entre la circulación intra y extracraneana al conectar el seno transverso con las venas occipitales, venas auriculares posteriores o venas cervicales profundas. Numerosas son las variantes descritas de este foramen acerca de su presencia, número, tamaño, ubicación y asimetría entre ambos lados. Su importancia quirúrgica radica en que pueden representar una fuente importante de sangrado, embolismo aéreo y sepsis, hechos que deben ser considerados por el cirujano al momento de realizar algún procedimiento o abordaje (Martínez et al., 2005).

La VEM tiene importantes funciones como enfriar la sangre y liberar la presión intracraneal cuando esta se encuentra elevada. Recientemente se ha utilizado como vía de acceso endovascular para el tratamiento de fístulas arteriovenosas dúrales (Rivet et al., 2006). Una característica importante de dichas venas es que no poseen válvulas (Chaunan et al., 2011), por lo tanto, la circulación puede ir en los dos sentidos de intracraneal a extracraneal y viceversa, sin embargo, la circulación tiende a ir en dirección hacia afuera del cráneo, ésta particularidad resulta de gran importancia ya que se asocia con diseminación de infección intracraneales cuando la VEM es lesionada (Louis et al., 2009).

La importancia de la VEM radica en que puede lesionarse y causar complicaciones serias en los abordajes neuroquirúrgicos de la fosa craneal posterior, del oído medio e interno (Cheatle, 1992). No encontramos estudios de la VEM y tampoco del FM que ésta imprime en el cráneo Louis et al.

Generalmente la VEM posee un calibre pequeño, su diámetro varía entre $0,8-4,5 \mathrm{~mm}$ y el diámetro del FM coincide con el calibre de dicha vena. Un FM se considera dilatado si su diámetro supera los $4,5 \mathrm{~mm}$ y se considera rudimentario si mide menos de $0,8 \mathrm{~mm}$ (Alsherhri et al., 2011).

Departamento de Anatomía Humana, Facultad de Medicina y Hospital Universitario “Dr. José Eleuterio González”, Universidad Autónoma de Nuevo León, Monterrey, Nuevo León, México.

** Departamento de Anatomía Humana, Facultad de Odontología, Universidad Autónoma de Nuevo León, Monterrey, Nuevo León, México. 
HERNÁNDEZ-RODRÍGUEZ, A. N.; GALINDO-DE LEÓN, S.; MORALES-AVALOS, R.; THERIOT-GIRÓN, M. C.; DE LA GARZA-CASTRO, O.; ELIZONDO-OMAÑA, R. E. \& GUZMÁN-LÓPEZ, S. Prevalencia y características morfométricas del foramen mastoideo y vena emisaria mastoidea en población Mexicana. Int. J. Morphol., 32(2):395-398, 2014.

Dicha característica adquiere gran relevancia al momento de una cirugía como las ya mencionadas debido al riesgo potencial de hemorragia si la VEM se encuentra dilatada y es lesionada. Para evitar esta y cualquier otra complicación lo recomendable es realizar una tomografía computarizada de alta resolución (TCAR) como método diagnóstico preoperatorio con el fin de visualizar si está presente o no ésta vena, determinar su diámetro y de esta manera evitar posibles complicaciones durante y después de la cirugía (Ahmad et al., 2011).

El estudio del FM permite conocer indirectamente las características de la VEM, ya que la presencia del FM en un cráneo seco se relaciona con la existencia de dicha vena. El presente estudio tuvo por objetivo determinar la prevalencia, variantes y características morfométricas del FM en población mexicana.

\section{MATERIAL Y MÉTODO}

Se realizó un estudio descriptivo, observacional de corte transversal en que se estudiaron las características morfométricas del FM.

Previo capacitación y estandarización, dos observadores independientes valoraron 88 cráneos secos (176 hemicráneos) pertenecientes a los Departamentos de Anatomía Humana de las Facultades de Medicina y Odontología de la UANL. Se determinó la prevalencia, variantes y características morfométricas del FM en ambos lados del cráneo. Las mediciones se realizaron utilizando un caliper Vernier digital (precisión de 0,01 mm) para la medición del ancho y largo de los forámenes mastoideos. Como fueron cráneos secos los que se examinaron, se consideró como referencia para verificar la prevalencia de la VEM el foramen que esta vena imprime en el cráneo en su ubicación más frecuente que se ubica en la sutura petromastoidea (Martínez et al.)

Se incluyeron todos los cráneos secos pertenecientes a los Departamentos de Anatomía Humana de las Faculta- des de Medicina y Odontología de la Universidad Autónoma de Nuevo León. Se excluyeron los cráneos con ausencia o daño estructural en el área de estudio.

Las mediciones de los forámenes fueron efectuadas con los cráneos en posición anatómica sobre una superficie plana. El largo del FM se determinó al evaluar la distancia entre el límite superior e inferior del foramen mastoideo trazando una línea imaginaria vertical, el ancho se delimitó por la diferencia entre el límite medial y el límite lateral del foramen trazando de la misma forma una línea imaginaria en dirección horizontal. Los observadores evaluaron ambos lados de los 88 cráneos y de esta manera se definió el número de forámenes existentes. Posteriormente se midió cada uno de los FM y se tomó como diámetro del mismo la medición del largo de cada foramen. Así mismo se obtuvo el promedio de las mediciones realizadas por los dos observadores cuyo resultado fue considerado el diámetro definitivo del FM, el cual se relaciona directamente con el diámetro de la VEM.

Se obtuvo un registro fotográfico de los cráneos estudiados (cámara profesional Canon EOS Digital Rebel XTi de 10.1 Megapixeles). Se determinó la prevalencia, variantes y características morfométricas del FM.

\section{RESULTADOS}

La prevalencia del FM en los 176 hemicraneos fue de un $75 \%$ (Tabla I). La prevalencia de forámenes encontrados dependiendo del lado del cráneo fue de un 76,13\% para el lado izquierdo, y un $72,86 \%$ para el lado derecho.

La mayor parte de los hemicraneos con foramen mastoideo presente $(\mathrm{n}=132)$, tuvieron un solo foramen $(81,82 \%)$, seguido un $16,67 \%$ con dos y sólo el $1,51 \%$ presentó tres forámenes (Tabla I).

Las características morfométricas del FM se reportan en la Tabla II. La media del largo de dicho foramen fue 2,42 mm (rango 1,03 - 5,43). La media del ancho del FM fue $1,80 \mathrm{~mm}$ (rango 0,81-3,57).

Tabla I. Frecuencia de foramen mastoideo y variaciones en el número por lado.

\begin{tabular}{lcccccc}
\hline & \multicolumn{2}{c}{$\begin{array}{c}\text { Lado Izquierdo } \\
(\mathbf{n = 8 8})\end{array}$} & \multicolumn{2}{c}{$\begin{array}{c}\text { Lado Derecho } \\
(\mathbf{n = 8 8})\end{array}$} & \multicolumn{2}{c}{$\begin{array}{c}\text { Total de hemicráneos } \\
\text { con foramen }(\mathbf{n = 1 7 6})\end{array}$} \\
\cline { 2 - 7 } & $\mathbf{n}$ & $\boldsymbol{\%}$ & $\mathbf{n}$ & $\boldsymbol{\%}$ & $\mathbf{n}$ & \% \\
\hline Ausencia de FM & 21 & 23,87 & 23 & 26,14 & 44 & 25 \\
Presencia de FM & 67 & 76,13 & 65 & 73,86 & 132 & 75 \\
1 FM & 55 & 82,08 & 53 & 81,54 & 108 & 81,82 \\
2 FM & 11 & 16,42 & 11 & 16,92 & 22 & 16,67 \\
3 FM & 1 & 1,5 & 1 & 1,54 & 2 & 1,51 \\
\hline
\end{tabular}


Tabla II. Características morfométricas del foramen mastoideo (valores en milímetros).

\begin{tabular}{lccccc}
\hline Parámetro & $\begin{array}{c}\text { Total de FM } \\
\text { encontrados }\end{array}$ & $\begin{array}{c}\text { Media } \\
(\mathbf{m m})\end{array}$ & DE & $\begin{array}{c}\text { Mínimo } \\
(\mathbf{m m})\end{array}$ & $\begin{array}{c}\text { Máximo } \\
(\mathbf{m m})\end{array}$ \\
\hline Largo & 158 & 2,42 & $\pm 0,814$ & 1.03 & 5,43 \\
Ancho & 158 & 1,80 & $\pm 0,571$ & 0.81 & 3,57 \\
\hline
\end{tabular}

En cuanto a los forámenes dilatados, se determinó como dilatado a cualquier foramen con un diámetro mayor a $4 \mathrm{~mm}$ y el porcentaje que se encontró con dichas características fue de un 5,06\%; En 150 forámenes mastoídeos $(94,94 \%)$ el diámetro fue menor a $4 \mathrm{~mm}$.

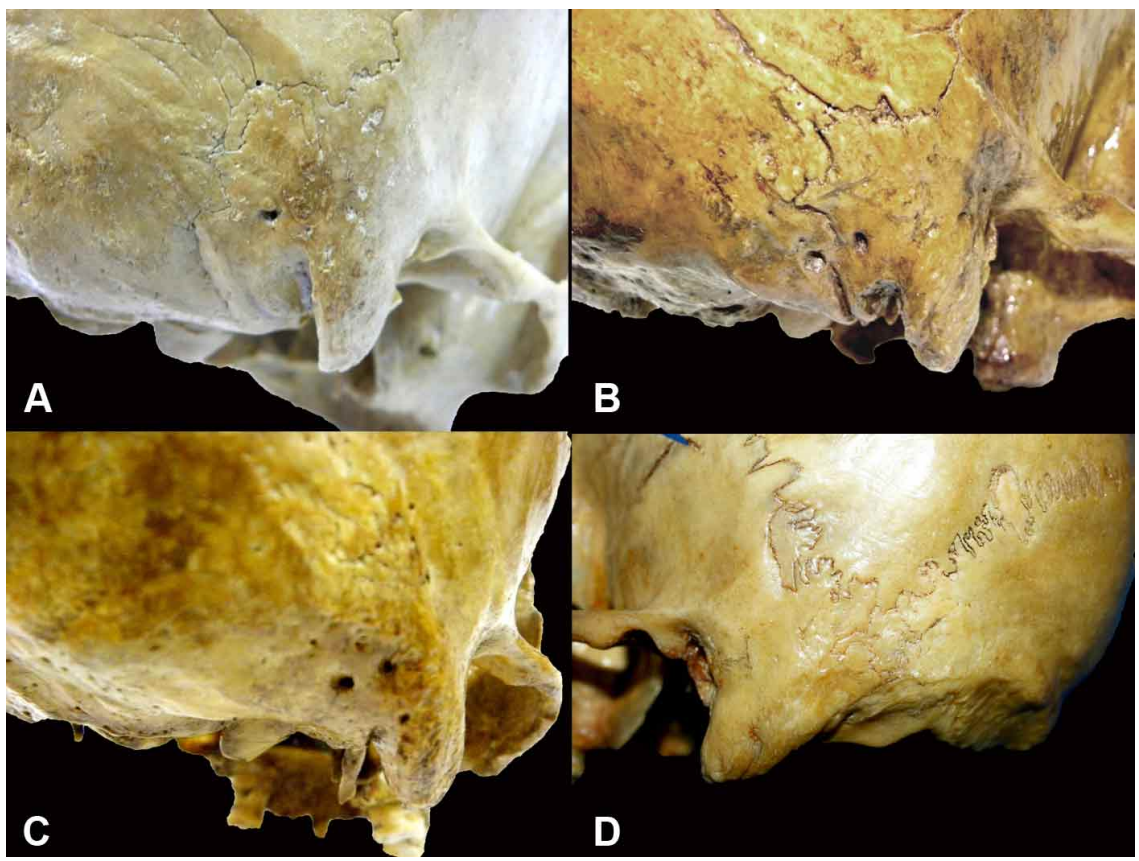

Fig. 1. Fotografías de una vista posterolateral de cráneos. A) Cráneo con un solo foramen mastoideo. B) Cráneo con dos forámenes mastoideos. C) Cráneo con tres forámenes mastoideos. D) Cráneo sin forámenes mastoideos.

\section{DISCUSIÓN}

Existe poca literatura acerca de la prevalencia, características morfométricas y variantes anatómicas del FM y la VEM. El conocimiento de la prevalencia del FM se relaciona con la existencia de la VEM. Los reportes encontrados señalan una frecuencia elevada, superior al $80 \%$ en poblaciones como la alemana en estudios realizados en diferentes años (Schelling, 1978; Koesling et al., 2005), en la población española (Martínez et al.) y norteamericana (Reis et al., 2007). Nuestro estudio es el primero realizado en población mexicana y se encontró una prevalencia del FM de 75\%, es uno de los porcentajes más bajos descritos en la literatura (Tabla III). Solo un estudio en población suiza (San Millán Ruíz et al., 2002) y el presente tienen frecuencias menores al $80 \%$.

Es importante conocer el número de FM presentes debido a posibles complicaciones quirúrgicas derivadas de su lesión. En el estudio de Louis et al., se encontró que es más frecuente tener más de un FM, en contraste con el nuestro donde se observó un FM más comúnmente. En el presente estudio encontramos que a mayor número de forámenes, disminuye su frecuencia de aparición.

Las características morfométricas del FM corresponden al diámetro de la VEM. Con dicho resultado se pudo diferenciar entre la VEM que es rudimentaria $(<0,8 \mathrm{~mm})$ y las que se encontraban dilatadas $(>4 \mathrm{~mm}$ ), dando como resultado una mayor precisión de la prevalencia de la VEM. El estudio de Reis et al., refiere que el diámetro promedio del FM fue de $2,15 \mathrm{~mm}$, lo cual coincide con el resultado de nuestro estudio. Sin embargo, el estudio presente determinó el alto y ancho del FM, por lo que su medición resulta mas precisa.

Los forámenes que miden más de $4 \mathrm{~mm}$ se consideraron dilatados, lo cual es importante porque implica un mayor riesgo de hemorragia de difícil manejo durante los abordajes de la fosa craneal posterior, además que puede ser indicio de alguna afección en la circulación intracraneal. En nuestro estudio encontramos una frecuencia de 5,06\% de FM dilatados. Esto coincide con los resultados observados en otros estudios (Ahmad et al.). 
HERNÁNDEZ-RODRÍGUEZ, A. N.; GALINDO-DE LEÓN, S.; MORALES-AVALOS, R.; THERIOT-GIRÓN, M. C.; DE LA GARZA-CASTRO, O.; ELIZONDO-OMAÑA, R. E. \& GUZMÁN-LÓPEZ, S. Prevalencia y características morfométricas del foramen mastoideo y vena emisaria mastoidea en población Mexicana. Int. J. Morphol., 32(2):395-398, 2014.

Tabla III. Prevalencia porcentual del foramen mastoideo en diferentes estudios.

\begin{tabular}{lcc}
\hline Estudio & Población & Frecuencia de FM \\
\hline Schelling et al. (1978) & Alemania & $80 \%$ \\
San Millan Ruiz et al. (2002) & Suiza & $63 \%$ \\
Louis et al. (2005) & USA & $85 \%$ \\
Martínez et al. $(2005)$ & España & $94 \%$ \\
Koesling et al. $(2005)$ & Alemania & $82 \%$ \\
Reis et al. (2007) & USA & $89 \%$ \\
Hernández et al. (2012) & México & $75 \%$ \\
\hline
\end{tabular}

AGRADECIMIENTOS. Al L. D. G Jaime Alberto Cisneros Ríos por su apoyo en cuanto a la imágenes gráficas y a todo el personal académico y administrativo que conforman los Departamentos de Anatomía Humana de las Facultades de Medicina y Odontología de la UANL y por las facilidades prestadas para el acceso a su material de osteoteca.

HERNÁNDEZ-RODRÍGUEZ, A. N.; GALINDO-DE LEÓN, S.; MORALES-AVALOS, R.; THERIOT-GIRÓN, M. C.; DE LA GARZA-CASTRO, O.; ELIZONDO-OMAÑA, R. E. \& GUZMÁN-LÓPEZ, S. Prevalence and morphometric characteristics of mastoid foramen and mastoid emmisary vein in the mexican population. Int. J. Morphol., 32(2):395-398, 2014.

SUMMARY: The anatomic descriptions of mastoid foramen (MF) and mastoid emissary vein (MEV) are lacking in the literature. This knowledge is fundamental for the surgeon who is about to perform surgery of the posterior fossa or mastoid region. This study aims to determine the prevalence of MF and MEV in Mexican population, determine the morphometric characteristics of MF and describe possible variations in the number, size, location and asymmetry. In this study 88 craniums were evaluated (176 hemicranium) by two independent observers who did not have access to information of each other's results. The prevalence, number and the morphometric characteristics were determined. MF prevalence of the two sides of the skull was $75 \%$ with a higher frequency of the left foramina compared to the right side; in reference to the number of foramina we found 108 hemicranium with a single MF, 22 double and 2 triple foramen. In conclusion, the prevalence of MF and MEV in this study is $75 \%$, this single variant noted as being the most frequent.

KEY WORDS: Mastoid foramen; Mastoid emissary vein; Prevalence; Morphometric characteristics; México.

\section{REFERENCIAS BIBLIOGRÁFICAS}

Ahmad, R.; Ali, I.; Naikoo, G. M.; Choo, N. A. \& Jan, F. Giant mastoid emissary vein: source of profuse bleeding during mastoid surgery. Indian J. Otolaryngol. Head Neck Surg., 63(Suppl. 1):102-3, 2011.

Alsherhri, H.; Alqahtani, B. \& Alqahtani, M. Dilated petrosquamosal sinus, mastoid emissary vein, and external jugular vein: A rare cause of pulsatile tinnitus, vertigo and sensorineural hearing loss. Indian J. Otol., 17(3):123-6, 2011.

Chauhan, N. S.; Sharma, Y. P.; Bhagra, T. \& Sud, B. Persistence of multiple emissary veins of posterior fossa with unusual origin of left petrosquamosal sinus from mastoid emissary. Surg. Radiol. Anat., 33(9):827-31, 2011.

Cheatle, A. H. The mastoid emissary vein and its surgical importance. Proc. R. Soc. Med., 18(Otol. Sect.):29-34, 1925.

Koesling, S.; Kunkel, P.; Schul, T. Vascular anomalies, sutures and small canals of the temporal bone on axial CT. Eur. J. Radiol., 54(3):335-43, 2005.

Louis, R. G. Jr.; Loukas, M.; Wartmann, C. T.; Tubbs, R. S.; Apaydin, N.; Gupta, A. A.; Spentzouris, G. \& Ysique, J. R. Clinical anatomy of the mastoid and occipital emissary veins in a large series. Surg. Radiol. Anat., 31(2):139-44, 2009.

Martínez, F.; Laxague, A; Vida, L.; Prinzo, H.; Sgarbi, N.; Soria, V. R. \& Bianchí, C. Anatomía topográfica del asterion. Neurocirugía, 16(5):441-6, 2005.
Reis, C. V.; Deshmukh, V.; Zabramski, J. M.; Crusius, M.; Desmukh, P.; Spetzler, R. F. \& Preul, M. C. Anatomy of the mastoid emissary vein and venous system of the posterior neck region: neurosurgical implications. Neurosurgery, 61(5 Suppl. 2):193-200, 2007.

Rivet, D. J.; Goddard, J. K. 3rd.; Rich, K. M. \& Derdeyn, C. P. Percutaneous transvenous embolization of a dural arteriovenous fistula through a mastoid emissary vein. Technical note. $J$. Neurosurg., 105(4):636-9, 2006.

San Millán Ruíz, D.; Gailloud, P.; Rüfenacht, D. A.; Delavelle, J.; Henry, F. \& Fasel, J. H. The craniocervical venous system in relation to cerebral venous drainage. AJNR Am. J. Neuroradiol., 23(9):15008, 2002.

Schelling, F. The emissaries of the human skull. Anat. Anz., 143(4):340$82,1978$.

Dirección para Correspondencia:

Dr. C. Rodrigo E. Elizondo Omaña

Departamento de Anatomía Humana

Facultad de Medicina

Universidad Autónoma de Nuevo León

Ave. Madero y Dr. Aguirre Pequeño s/n

Col. Mitras Centro, C.P. 64460

Monterrey, Nuevo León

MÉXICO

Email: rod_omana@yahoo.com

Recibido : 28-12-2013

Aceptado: 06-02-2014 